\title{
Designing the Metaverse
}

\author{
Stefan Seidel \\ University of Liechtenstein \\ stefan.seidel@uni.li \\ Gregory Yepes \\ Electronic Arts \\ gyepes@ea.com
}

\author{
Nicholas Berente \\ University of Notre Dame \\ nberente@nd.edu \\ Jeffrey V. Nickerson \\ Stevens Institute of Technology \\ jnickers@stevens.edu
}

\begin{abstract}
The Metaverse, a term coined in science fiction, is now being discussed seriously as a new form of infrastructure. The Metaverse is intended to make possible thematically interconnected immersive experiences. In this paper, we conceptualize the Metaverse as a meta design space. Within this space, designers create various interconnected design spaces. We highlight how the key dimensions of human experience (time, space, actors, and artifacts) each introduce tensions for making decisions in those design spaces, and we highlight the transitions between design spaces. This conceptual language opens up this novel and emergent phenomenon both to those wishing to design new disruptive technologies and those seeking to improve existing platform strategies. We conclude by highlighting how the Metaverse will not only comprise immersive virtual experiences but also transitions between physical and virtual experiences.
\end{abstract}

\section{The Metaverse: interconnected, immer- sive social experiences}

So Hiro's not actually here at all. He's in a computer-generated universe that his computer is drawing onto his goggles and pumping into his earphones. In the lingo, this imaginary place is known as the Metaverse. Hiro spends a lot of time in the Metaverse. It beats the shit out of the $U$ Stor-It.

Neal Stephenson, Snow Crash [1]

Hiro is the protagonist in Neal Stephenson's science fiction novel Snow Crash, originally published in 1992. He is a pizza delivery boy, programmer, and he collects information for the CIC (a private successor to the CIA). The setting is a future dystopia where society is run by the mafia. Hiro-along with many others-escape the dreadful situation through the
Metaverse - a large-scale virtual world that they populate through avatars.

The term "metaverse" has captured the imagination of the gaming industry for more than a decade. Recently, in 2021, Tim Sweeney, founder of Epic Games, claimed that it is important that the Metaverse (singular, capital ' $\mathrm{M}$ '!) will not be controlled by any corporation, but in fact by anybody [2]. He further calls Epic's most popular product (Fortnite) a metaverse, not a game. While the concept of metaverse has been subject of science fiction for some time, we are now starting to see manifestations beginning to emerge as virtual contexts for entertainment, social interaction, and commerce. From this point on, we will use the singular form with capital ' $M$ ' to highlight the potentially pervasive nature of the concept.

We define the Metaverse as the networks of digital technologies and people providing immersive, interconnected experiences. We further conceptualize the Metaverse as a meta design space, where Metaverse designers create various interconnected design spaces, each of which creates unique experiences.

These experiences are synthetic but also sensorially rich. These experiences are also composable: one can follow another, and they can potentially be tied together in a coherent manner, as in a well-choreographed travel experience, or a simulated repair movie overlayed on a physical appliance. These experiences are facilitated by computers and can be seen as another step in what Andy Clark sees as the inevitable merger of technology and biology [3].

In this sense, we can think of the design of the Metaverse as the design of an infrastructure upon which experiences can be interconnected. This view is consistent with the view of information infrastructure as being a higher construct above platforms and platform ecosystems [4]. As Hanseth and Lyytinen note, infrastructures are hard to design top down, since their control is usually distributed [4]. They often emerge as the result of negotiation. This negotiation can take the 
form of a conflict and resulting compromise among actors with different goals [5]. The Metaverse infrastructure will need to involve not only interoperability standards but also running systems. Every virtual experience will need to render data into sensory input, and this might involve a great deal of computation to represent the same state to large number of people in a shared experience. Moreover, movement across experience clusters, the universes of the Metaverse, might involve translations of currencies, artifacts, and ontologies. While one potential future involves a Metaverse constituted in a walled garden run by a technology company, another future involves a fully distributed infrastructure, along the lines of, but more general than the burgeoning area of decentralized finance and related distributed ledger schemes [6].

The Metaverse is a distinct socio-technical phenomenon [7] that now exists in different academic and popular discourses. First, it is built on the complex technology stack that we call the Internet and requires advanced computational capabilities to visualize complex 3D worlds. Second, these worlds need to fit a likewise complex social system that participates and actively shapes this world. The objectives - both instrumental and humanistic - cannot be defined in terms of conventional information systems. Conventional information systems represent some real-world phenomenon, augment a physical device, or constitute a game where some player pursues some goal. The Metaverse builds on the confluence and integration of varied immersive social experiences that move beyond the physical limitations of what we commonly perceive to be the real world, and it may play an important role for a variety of social activities and economic transactions [8-10]. It is not simply the next generation of video games or a new type of virtual reality. The Metaverse reflects ideas such as a-teleological systems [11] and highlights emergence, evolution, and open-endedness. This is contrary to common information systems that typically meet some specific - mostly instrumentalpurpose and have confined boundaries. Designing for the Metaverse means designing for open-endedness, emergence, and experiences that transcend established boundaries. It is much more akin to remixing, with an emphasis on recombination and the generation of variation [12] than it is to optimization, which emphasizes convergence to a particular outcome.

Therefore, if an organization or an individual were to attempt to start strategically positioning itself for this future Metaverse, it is important to think through how this dynamic context may be structured. In this paper, it is our intention to provide conceptual foundations that open up this novel and emergent phenomenon for thinking about the Metaverse from a design and strategic perspective. At its core, the
Metaverse is about providing interoperation between diverse platform ecosystems. We can broadly conceive of software platforms as "the extensible codebase of a software-based system that provides core functionalities shared by the modules that interoperate with it and the interfaces through which they interoperate" [13, p. 675]. Beyond particular platforms are platform-based ecosystems that are the sum of the platform and the modules that are available through that platform. Platform ecosystems facilitate interactions among distinct groups, such as developers of modules and users of those modules [14]. The Metaverse, as an integrator of these ecosystems, will have as stakeholders some groups who will provide experiences, others who will consume experiences, and others who will do both. Many of these stakeholders are actively attempting to define standards. Because of the unpredictable, remixed and reformulated, experience-based quality of the Metaverse, standardization will be important, but it is unlikely that current standards efforts will adequately define this complex, emergent, experiential landscape.

We proceed as follows. The next section describes some Metaverse foundations, highlighting how the term has been used in different fields and from different perspectives, but where the key reoccurring elements are experience and interconnectedness. We then turn to the key dimensions of human experiences (space, time, actors, and artifacts) that, together with fundamental ideas from the sciences of the artificial (design as problem solving, modularization to manage complexity and to allow for emergence), provide the conceptual foundation for our development. Our conceptual development suggests that the Metaverse is a meta design space (from the perspective of the designer role) and a meta experience space (from the perspective of the user role) allowing for the integration and interconnection of various design spaces and associated design experiences. We discuss our conceptualization in relation to platform ecosystems and standards as well as layered modular architectures, and we conclude by sketching how an artificially intelligent architecture might enable this eventual ecosystem of platform ecosystems.

\section{Metaverse foundations}

Dionisio and colleagues [9] describe the Metaverse as moving "from a set of independent virtual worlds to an integrated network of 3D virtual worlds" [p. 34:2] that "constitutes a compelling alternative realm for human sociocultural interaction" [p. 34:2]. These authors further highlight how building the Metaverse rests on developments in four key areas, namely (1) immersive realism, (2) ubiquity of access 
and identity; (3) interoperability; and (4) scalability. They focus on the current state of computing to enable the Metaverse. Other definitions have applied the notion to interconnected mixed reality spaces [8] or to learning in virtual spaces $[10,15]$.

There are two key constituting aspects that together differentiate the Metaverse from other phenomena that also create virtual experiences, such as video games and virtual worlds. First, the Metaverse integrates and interconnects varied social experiences in one encompassing system. Second, the Metaverse does this without the need for game-specific rules and goals. That is, the Metaverse provides a digital infrastructure $[4,16]$ that allows a wide range of experiences to be composed into a whole. Humans perform a composition of experiences when they plan an evening out, based on the infrastructure of a city, and the Metaverse will involve a similar infrastructure for humans to enact experiences. In contrast, however, it is important to remember that the Metaverse has virtual, synthetic aspects and therefore allows for various activities that do not have to adhere to physical limitations. The Metaverse allows its "residents the ability to fly, to teleport, to change gender or even adopt nonhuman forms and indeed the ability to switch back and forth between these different persona" [15]. The Metaverse provides alternate human experiences that move beyond the boundaries of the real world, augmented or not.

This is hinted by recent developments in gaming environments. For example, Epic Games launched a violence free island within their game Fortnite to host a series of concerts. Qualitatively different experiences - including different rules_-were made available to human agents in the same digital space. This notion of experience and transition among experiences will be a key defining feature of the Metaverse.

Moreover, there are some practical emerging areas of design. For example, human drivers in level 4 autonomous vehicles may be engaged in virtual worlds when the vehicle finds itself in a situation that requires human control: the vehicle will be summoning the driver out of virtual reality and the driver will need to transition, acquiring situational awareness. The summoning and the transition are both aspects of the Metaverse.

In the next section, we examine the key dimensions of human experience used to study how digital technologies have revamped everyday life experiences, to then apply this conceptual framework to the analysis of the Metaverse from a distinct design perspective.

\section{Computing and experience}

Recent information systems literature has highlighted the reality-shaping nature of digital technologies [17-19]. One key aspect in this reality-shaping nature of digital technologies is how they relate to human experience [20]. Key dimensions of human experience have been discussed in the context of experiential computing - the phenomena related to how human experience is increasingly penetrated by, and intertwined with, computing technology [20]. Humans use digital technologies in everyday tasks - tasks that are not computational per se, such as talking or shoppingand digital technologies mediate the various dimensions of human experience (Figure 1).

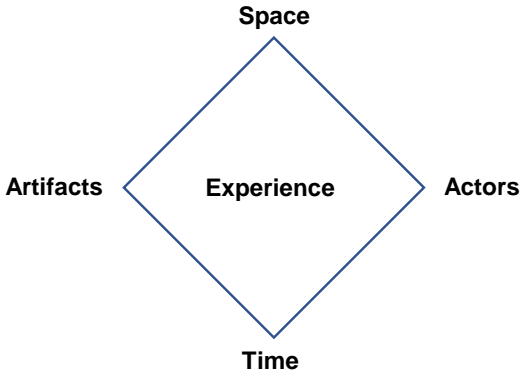

Figure 1. Key dimensions of human experiences [20]

While experiential computing attends to experiences in a physical world penetrated by digital technologies, the Metaverse creates experiences in an alternate reality - an advanced form (both qualitatively and quantitatively) of imagined computing [20]. Next to experience, however, one key element of the Metaverse is the interconnectedness of experience-it is the integration of various experiences [9].

Next, we use these four dimensions of human experience to attend to the key design decisions about the Metaverse as varied and interconnected immersive experiences with unprecedented depth. We add a fifth dimension that is central to the idea of the Metaverse as a meta design space - the transition dimension, which conceptualizes the integration of different design spaces and the movement between those design spaces.

\section{The Metaverse: Design spaces and de- sign tensions}

Creating the Metaverse is a design problem where designers (1) identify and implement feasible solutions [21] to create distinct experiences and (2) understand these as part of a meta-design space that allows for the integration of separate experiences. We capture 
the first aspect in the key dimensions of human experience and the second in an additional transition dimension. That is, each unique experience is a design problem itself, but so is the overall design of the Metaverse.

The various design dimensions vary along their dimensional ranges, and thus involve tensions-spatial tensions, temporal tensions, artifactual tensions, actor tensions, and transitional tensions. Together, these dimensions constitute the overall problem space in which feasible solutions can be generated, each describing a distinct experience space.

Figure 2 visualizes the Metaverse as a meta design space of interconnected design spaces. While each design space creates unique experiences through variations along artifactual, spatial, temporal, and actor dimensions, it is the meta design space that defines important aspects such as the Metaverse's overall governance, including questions of control and ownership. Tensions that may exist across the various dimensions of human experience thus exist in various combinations, which reflect the designers' specific design choices.

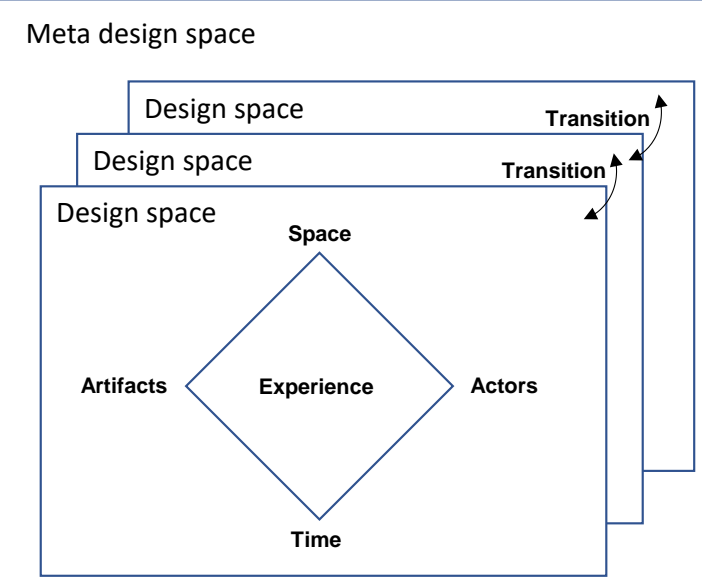

Figure 2: The Metaverse as a meta design space of design spaces

\section{Spatial tensions (where)}

Human experience - in the real world as in alternate realities created by digital technologies - exists in space. While early computer games, for instance, provided two-dimensional experiences, contemporary video games mostly present vast three-dimensional spaces. Limitations that exist in the physical world do not exist in the digital world, at least not conceptually, and contemporary computer games are free to vary physical constants [22]. In Neal Stephenson's novel, we read the following about the grand street of the Metaverse in which Hiro immerses himself:

The dimensions of the Street are fixed by a protocol, hammered out by the computer-graphics ninja overlords of the Association for Computing Machinery's Global Multimedia Protocol Group. The Street seems to be a grand boulevard going all the way around the equator of a black sphere with a radius of a bit more than ten thousand kilometers. That makes it 65,536 kilometers around, which is considerably bigger than Earth.

Key spatial design decisions in the Metaverse are related to whether-and how-the Metaverse constrains versus affords movement in space. To conceptually attend to these tensions (spatial constraint versus spatial affordance), we can draw on some terminology that is used in video game development, where the notion of open world describes virtual spaces that allow users free navigation in an open space. An open world affords users to freely navigate the Metaverse (or the part of the Metaverse she is currently in), while a closed world approach (deliberately) constrains this navigation - that is, the Metaverse designer can decide on the spatiality of experience. Figure 3 illustrates this view.

\section{Closed \\ Open-ended}

Figure 3: Designing Metaverse spatiality

As the Metaverse combines varied experiences, it can combine all flavors of closed and open-world approaches. We see some evidence of that, for instance, in Epic's Fortnite, where parts of the game provide an open-world experience while the aforementioned concert provides a limited space where users attend that concert.

\section{Temporal tensions (when)}

Human experience exists in time. Most conceptions of the Metaverse assume that experience is in real-time (opposed to asynchronous), but the Metaverse designer can decide on the temporality of experience. Consider what Neal Stephenson writes in Snow Crash:

A Metaverse vehicle can be as fast and nimble as a quark. There's no physics to worry about, no constraints on acceleration, no air resistance. Tires never squeal and brakes never lock up. The one thing that can't be helped is the reaction time of the user. So when they were racing their latest motorcycle software, holding wild rallies through Downtown at Mach 1, they didn't worry about engine capacity. They worried about the user interface, the controls that enabled the rider to transfer his reactions into the machine, to steer, accelerate, or brake as quickly as he could think. 
We can think of experiences that are contemplative, that take place at slow pace, where every movement takes a considerable amount of time-perhaps more than compared to what we would expect in real life. We can also think of experiences that are reactive, fast-paced, perhaps more reactive than what we normally experience in the real world. A 3D chess game might be contemplative, a shooter might be reactive, and a social experience in the Metaverse might have elements of both. Different genres will require different pace and hence different experience temporality. Figure 4 illustrates this view.

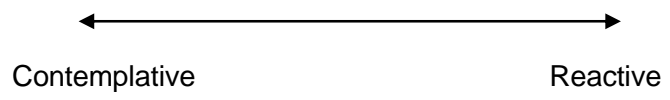

Figure 4:Designing Metaverse temporality

Designers of each experience will have to make design decisions along the dimension of temporality. Given the reactive nature of many games, one might expect this dimension to dominate, but there are surprising number of contemplative activities, including puzzle-solving, sculpting, and meditation, represented in the current repertoire of virtual reality games. Moreover, the contemplative activities may have health benefits [23].

\section{Artifactual tensions (what)}

Humans experience artifacts as they perceive them and interact with them, both in the real world and the Metaverse. While artifacts in traditional information systems are representational in that they provide a high-fidelity representation of some focal realworld phenomenon $[24,25]$, this is not necessarily the case in the Metaverse. Artifacts can be representational (such as when a rock is displayed that is based on a real-world rock), but they can also be imagined (dragons do not exist in the real world).

Recent technical advancements allow for detailed-high resolution images - but what people want is often fidelity. Fidelity is a measurement of the quality at which a certain artifact is a representation of some focal phenomenon. Metaverse designers may deliberately depart from any real-world experience and provide imagery that cannot be thought of as a faithful replication of an existing location. Hiro experiences the grand street of the Metaverse as follows:

It is the Broadway, the Champs Elysees of the Metaverse. It is the brilliantly lit boulevard that can be seen, miniaturized and backward, reflected in the lenses of his goggles. It does not really exist. But right now, millions of people are walking up and down it.
Still, it might be an oversimplification to see "imagined" as the opposite of "faithful." Instead, we can apply the concept of fidelity to the concept of imagination - if something imagined is displayed with a high level of detail it seems reasonable to suggest that it is a high-fidelity representation of an imagination.

This becomes clear when we consider how much of what we are seeing in, for instance, contemporary video games are imagined versions of high-fidelity representations - for instance rocks that look like real rocks but do not necessarily have real-world counterparts (no specific rock exists in the real world that is the 'original' for that specific representation).

We can thus define artifact characteristics in the Metaverse in terms of fidelity and imagination (Figure 5).

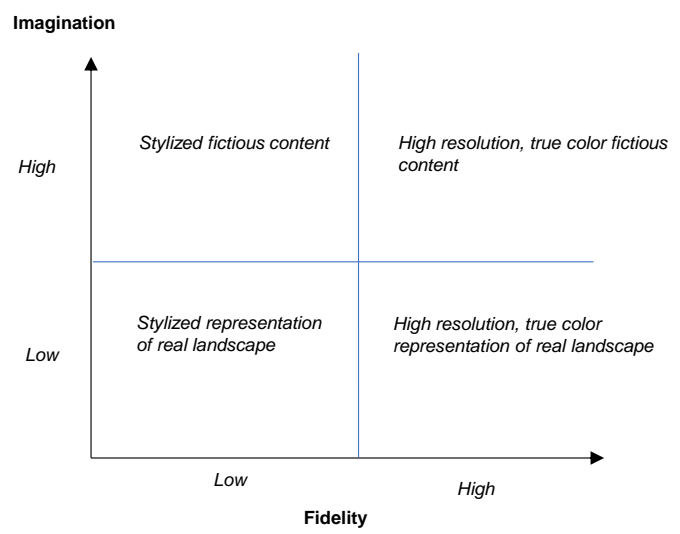

Figure 5: Designing Metaverse artifacts

\section{Actor tensions (who)}

To experience the Metaverse, users need some form of digital interface. Contemporary versions, like the one proposed by Epic Games, use third-person or first-person avatars. These have specific properties and capabilities to interact with the Metaverse's artifacts as well as other actors across the Metaverse's space and time. Hiro has chosen to just look like Hiro in the Metaverse:

Hiro's avatar just looks like Hiro, with the difference that no matter what Hiro is wearing in Reality, his avatar always wears a black leather kimono.

The Metaverse designer can decide whether the actor is in resemblance to the human user (on social media sites humans represent themselves) or whether the user can take on a very different role, perhaps coupled with different capabilities (a concept known from role-playing games). In the physical world there is a singular self, and although that self can have a variety of roles and identities, there is, in the end, one physical 
instantiation. In the Metaverse, however, the singular, physical self can generate multiple instantiations (or entities, or avatars, or personas). These entities can be entirely unrelated, or, in some instances they can be linked. Although the individual (non-virtual) self may only experience the Metaverse when actively engaged, the online selves may be active and working toward some goals or influencing environments in some way. In this sense, the singular self may delegate to multiple selves, and the multiple may act, to some degree, autonomously [26]. Figure 6 visualizes this view.

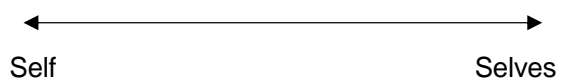

Figure 6: Designing Metaverse actors

\section{Transition tensions}

Experience dimensions describe the problem space in which designers create unique experiences within the Metaverse. The transition dimension (and associated tensions) describes the integration of different experiences (and hence design spaces) across the Metaverse - that is, how one moves from one experience to another. In Snow Crash's Metaverse, one gets around using a monorail:

It is a hundred meters wide, with a narrow monorail track running down the middle. The monorail is a free piece of public utility software that enables users to change their location on the Street rapidly and smoothly. A lot of people just ride back and forth on it, looking at the sights.

From the perspective of the Metaverse as a meta design space, another question is: How does one move from one experience to another, that is, from one design space to another. While the first four design dimensions addressed experience, this fifth design dimension addresses interconnectedness.

To describe a transition, we require three elements: the source, the destination, and the transformations required to move from one experience to the other.

The transition dimension is thus orthogonal to the other four design dimensions as any transition applies to spatiality, temporality, artifacts, and the actor. Actor transitions, for instance, may involve a change in the user's roles and abilities. Spatial transitions may involve moving from a two-dimensional space into a three-dimensional space. Temporal transitions may change the pace of interaction. Artifactual transitions may involve moving from one environment to another, perhaps with entirely different physics and applicable rules.
We can thus describe two elements of transitions that relate to (1) the experience (artifacts, space, temporality, actors) as one moves from one space to another, and (2) the design of the transformation itself. Regarding the experience, transitions may vary from incoherent to coherent: if the transition involves moving from one space to another, where each space creates quite different experiences, the transition is incoherent (for instance, as one moves from a two-dimensional game to a three-dimensional game or from a shooter experience to a racing experience). Coherence has been measured in relation to both texts and images [27, 28].

Regarding the transformation, it can vary from discontinuous to continuous: a continuous transition is one where the transformation occurs in a stepwise manner, whereas in discontinuous transformation the transition is abrupt. Figure 7 visualizes this view.

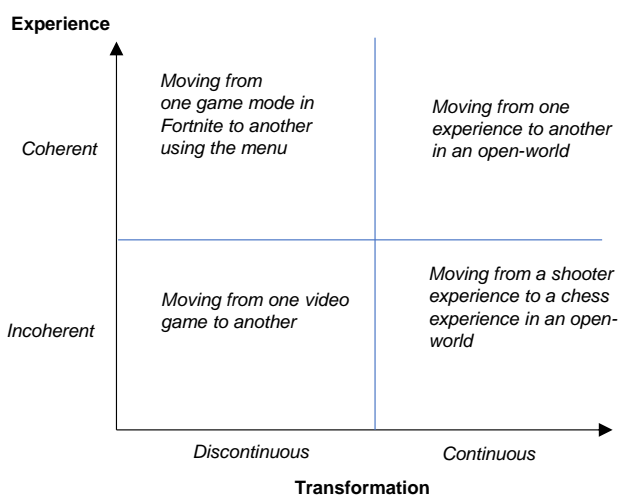

Figure 7: Designing Metaverse transitions

Consider Fortnite, where the gamer can move between different experiences, but where the transition from one experience to another is through a user interface. On the one hand, the same engine is used for all experiences and the physics, and looks are comparable, thus creating a sense of coherence. On the other hand, the user must use a user interface, that is, must leave one experience to move to another, creating a sense of discontinuity.

The notion of coherence is important to the composition of experiences. For example, one can plan a night out where the events seem discrete, or one can plan a night out with themes that surface in each of the experiences. Indeed, one of the important aspects of narrative construction is the introduction of connecting tissue between episodes that on the surface are dissimilar. For example, several episodes might be connected by a common activity: back-to-back episodes in Paris, Berlin, and London might explore virtual art 
scenes. Just as in literature, connecting tissue might involve the use of metaphor: for example, a shovel in a construction game might morph into a wand in a phantasy game.

Cities allow for fun evenings because experiences can be composed in coherent ways. Because city infrastructure creates a dense landscape of services, many experiences can be combined in a short amount of time [29].

The Metaverse could also allow experiences to be composed in coherent ways. Whereas a city relies on physical zoning and transportation infrastructure, the Metaverse might rely on virtual zoning and scene-transition infrastructure. Even though one can imagine a randomly created graph of virtual services, there are advantages to some kind of virtual zoning in which conceptually close experiences are close to each other. And even though transitions might be designed to be instant, slower transitions might be important for several reasons. Cognitively, we are used to adjusting to a new context even before we enter it, and so some time to adjust may be important. Technically, moving from one virtual experience to another may mean rebuilding complex high-fidelity maps while converting currencies and artifacts for use in a new context. Some time to get that done may be necessary to avoid waits and glitches.

More generally, to be effective, the Metaverse will want to host many different experiences that can be recombined. The meta experience - the virtual night out - can then be designed, and coherence for most would be one of the design objectives.

\section{Discussion}

The dimensions we have introduced in this paper are intended to help scholars theorize about the Metaverse. They are not intended to be comprehensive. In this section, we discuss how the emergent discourse on the Metaverse has its heritage in established discourses in the Information Systems field - and how the discourse on the Metaverse can draw on those discourses as well as contribute to those discourses.

We can view the development of the Metaverse in the trajectory of computation in the digital age. For decades, digital technologies have been interwoven with the fabric of organizations [30], building on their key capability to represent some focal real-world phenomenon such as a system or process [24, 25] —also referred to as representational computing [20]. Over the past two decades or so, digital technologies have become part of our everyday experiences, in terms of digitally-mediated embodied experiences (such as when people use wearables or navigate with their
iPhones) - also referred to as experiential computing [20]. A third category is imagined computing, where the interaction with a computer is an end in itself, as in the case of video games or virtual worlds [20].

Continuing from this view, the Metaverse moves beyond merely representing or augmenting the real world but aims to generate alternate realities - as foreseen in Neal Stephenson's novel. As it involves interactivity and sociality and as it interconnects a broad variety of human experiences, it moves beyond a simple notion of imagined computing and foreshadows another step in the evolution of computing - transcendental computing. That is, computing that allows for new categories of experience. This transcendence will rely on a technical stack rooted in Internet technologies. And the technical stack may need to be extended.

\subsection{Platform ecosystems and standards}

One way to think about the Metaverse is as a collection of universes, such as game universes (as indicated earlier, we can conceive of universes as the experience clusters of the Metaverse). Every universe has a set of services that allow for the persistence of past experience, reputation, found artifacts, and purchased artifacts or skins. That is, every universe has an infrastructure. The Metaverse encompasses these different infrastructures, and services it provides include ways of translating or converting currencies or skills or artifacts from one universe into another. Many of the universes think of themselves as platform ecosystems, in that they may allow for user-based content and third-party sellers who provide additional goods and services. Some researchers have begun talking about an ecosystem of ecosystems [31]. Maybe this climb up in abstraction is necessary. Or maybe the process of building the Metaverse will result in more streamlined and loosely coupled architectures.

One place to look for theories that might guide the study of the Metaverse is organizational design. Mintzberg, in discussing corporations, made the general observation that there are five coordinating mechanisms between entities. One is mutual adjustment, and another is direct supervision. The next three are types of standardization: Standardization of work processes, standardization of outputs, and standardization of skills. He points out that it is standardization that can relax the need for direct supervision, while at the same time reducing the need for mutual adjustment [32]. Indeed, information systems literature has engaged with how technological standards emerge in ecosystems [32], and of late, standardization is an active area of research for various services expected of the Metaverse [9, 33]. 
In one view, the meta design space provides the foundation for various design spaces. Each design space - and hence each experience - is a platform for content and actors. While the meta design space provides the rules that apply to everything that exists in the Metaverse, each design space will have its own rules to create distinct experiences. In this view, the meta design space is an infrastructure that constrains the design choices for all subordinate design spaces and hence platform ecosystems that exist in the Metaverse.

Platforms can create external network effects [34]. Indeed, the Metaverse requires such effects to be sustainable [9]: the Metaverse needs to push for universality, since otherwise, there will not be enough recombination possible to make the extra level of hierarchy valuable.

Another perspective is the following. If the Metaverse creates strong standardization around services, then perhaps the boundaries of current universes, such as game universes, will be increasingly artificial. That is, maybe just one level is needed, but a level that is truly universal. This way of thinking draws from the experience of Internet standardization, which purposefully pushed for universal mechanisms [35]. This would suggest that the boundaries between the various platform ecosystems that make up the Metaverse will increasingly diminish.

Specifically, Internet standards provide modularity. On this view, various interconnected experience spaces as well as the various elements in those spaces are modules. The Metaverse platform then provides control and coordination and orchestrates the modules that are available through the platform [34].

While modularity is generally desirable by all potential Metaverse stakeholders, for the larger companies, walled gardens usually provide more profit than open parks. So many companies are willing to modularize services, but within a walled garden. We can already find some Metaverse elements in existing platform ecosystems. For instance, being in the Apple ecosystem allows users to participate in a variety of experiences (listening to music, watching videos, using a variety of apps) and transitioning between these experiences is relatively easy, albeit not continuous. Is there a world where the walled garden owners are willing to release some of their control, under the belief that they will prosper from growing interest? The recent position statements and legal fights involving these owners - including Apple, Epic, Facebook, and Google - presage negotiations to come [36]. In particular, the dimensions of the meta design space may affect the platforms: increased combinatorics may increase participation, while at the same time creating long tails of personalized experiences. Transitions may require sharing of computational resources, which may encourage cross-platform bundling to decrease the complexities of billing users or competitors.

\subsection{Digital innovation \& layered modular ar- chitectures}

We have conceptualized the Metaverse as interconnected design spaces aiming at generating various interconnected experiences, and we have highlighted how the Metaverse can provide the foundation for various experiences. In this sense, the Metaverse can be generative by facilitating recombination [37].

In digital innovation, "digital technologies and associated digitizing processes form an innate part of the new idea and/or its development, diffusion, or assimilation" [38, p. 224]. A key factor allowing for digital innovation is the organization of digital artifacts in a layered modular architecture, which is a key organizing principle of the contemporary Internet [20].

First, modularization allows for fast growth and emergence [39]. Second, the organization in different layers allows Metaverse participants to be innovators at different levels. While companies like Nvidia may be providing the devices that are required to power the immersive visualization of the Metaverse, game developers like Epic may provide the engines that are required to generate that immersive visualization.

Of course, these are generally for-profit companies, and they look to leverage successful modules by subsuming related modules in their efforts to expand their platforms - a practice referred to as envelopment [40]. The Metaverse will involve emergent experiences in an open-ended, layered modular platform architecture. New, emergent experiences will continuously emerge as existing domains are continually contested.

\subsection{Artificial intelligence}

Because of the sheer scope, scale, and unpredictable, emergent character of the Metaverse, it is a likely candidate for the incorporation of artificially intelligent technologies. Such technologies will, of course, be involved with the experiences themselves, but also with the structuring of the transitions and the underlying standards.

In terms of experience, the vast amount of content needs to be available and interactive. Such vast amounts of content cannot be generated by human designers alone. Instead, increasingly autonomous tools powered by artificial intelligence methods will be able to generate high fidelity content with speed and at scale [41]. 
In terms of standards, one cannot predict the use cases for between-environment experiences - the transitions, translations, and transformations that will make the Metaverse what it will become. There will be a host of opportunities for artificially intelligent agents to play different roles that support new kinds of experience. Such agents need to learn, reconcile, and enact the delegated agency of those interacting with the Metaverse. They will act as the procedural glue that can integrate experiences. If we take contemporary video games and online environments as the reference point, non-human characters and bots will be implemented in terms of rational, perhaps learning, agents that can evolve as they adjust their behavior over time [42].

The use of artificial intelligence for content generation and to support emergent experiences is critical for any organizational strategy that involves the Metaverse.

\section{Conclusion}

The Metaverse can be viewed as the next generation of the Internet that increasingly provides varied and interconnected immersive experiences. To this end, we have conceptualized the Metaverse as a meta design space that supports transitions and recombination of experiences centered in current and future platform ecosystems.

The Metaverse is now an empirical phenomenon as software vendors aim to move towards implementing such interconnected immersive experience and have started to create business models around the idea. Moreover, the tussle over who will own the Metaverse - and thus questions of ownership, power, control, and governance that move beyond mere technical issues-has started.

One may argue that the Metaverse discussion is old wine in new bottles and that similar discussions have been led in the context of immersive systems and virtual worlds. However, the universality of the Metaverse concept suggests that the Metaverse might be the next evolutionary stage of the Internet, as it allows for the recombination not just of web content, but of immersive and emergent experiences. Moreover, the Metaverse will involve not only transitions between immersive virtual experiences but transitions between physical and virtual experiences. Simulations allow us to create things in a virtual world that are then transformed back into physical artifacts and processes, thus increasing our overall ability to produce goods and services. The thrust for integration and interconnectedness of tomorrow is not going to look the same as yesterday.
The confluence of platform ecosystems and artificial intelligence will work to provide a variety of experiences. The information systems design challenges revolve around the question of how to recombine these experiences. If we conceive the Metaverse to be an evolved version of the Internet, then we are led to consider the large-scale societal consequences of this technology: Who will use the Metaverse? Who will own the Metaverse? And, most importantly, to what end?

\section{Acknowledgements}

This material is based upon work supported by the National Science Foundation under grants 2128906, 2113906 , and 1909803.

\section{References}

[1] N. Stephenson, Snow Crash: A Novel. Spectra, 2003.

[2] T. Sweeney, "Why We Need an Opern Metaverse," D. Takahashi, Ed., ed, 2021.

[3] A. Clark, Natural-born Cyborgs: Minds, Technologies, and the Future of Human Intelligence. Oxford University Press, 2003.

[4] O. Hanseth and K. Lyytinen, "Design Theory for Dynamic Complexity in Information Infrastructures: The Case of Building Internet," Journal of Information Technology, vol. 25, no. 1, pp. 1-19, 2010.

[5] D. D. Clark, J. Wroclawski, K. R. Sollins, and R. Braden, "Tussle in Cyberspace: Defining Tomorrow's Internet," IEEE/ACM Transactions on Networking, vol. 13, no. 3, pp. 462-475, 2005.

[6] H. Chen, E. Gu, and Y. Jiang, "Metaverse: The New Reality," 2018. [Online]. Available: https://metaverse.nyc3.digitaloceanspaces.com/whitepaper/Metaverse-whitepaper-v3.0-EN.pdf

[7] S. Sarker, S. Chatterjee, X. Xiao, and A. Elbanna, "The Sociotechnical Axis of Cohesion for the IS Discipline: Its Historical Legacy and its Continued Relevance," MIS Quarterly, vol. 43, no. 3, pp. 695-720, 2019.

[8] B. Ryskeldiev, Y. Ochiai, M. Cohen, and J. Herder, "Distributed Metaverse: Creating Decentralized Blockchain-based Model for Peer-to-peer Sharing of Virtual Spaces for Mixed Reality Applications," in Proceedings of the 9th Augmented Human International Conference, 2018, pp. 1-3.

[9] J. D. N. Dionisio, W. G. B. III, and R. Gilbert, "3D Virtual Worlds and the Metaverse: Current Status and $\mathrm{Fu}-$ ture Possibilities," ACM Computing Surveys (CSUR), vol. 45, no. 3, pp. 1-38, 2013.

[10] D. M. Barry et al., "International Comparison for Problem Based Learning in Metaverse," The ICEE and ICEER, vol. 6066, 2009.

[11] L. D. Introna, "Notes on Ateleological Information Systems Development," Information Technology \& People, vol. 9, no. 4, pp. 20-39, 1996. 
[12] J. V. Nickerson, "Remixing Systems: Collective Design through Modification," in Handbook of Digital Innovation, S. Nambisan, K. Lyytinen, and Y. Yoo Eds.: Edward Elgar Publishing, 2020, pp. 133-149.

[13] A. Tiwana, B. Konsynski, and A. A. Bush, "Research Commentary-Platform Evolution: Coevolution of Platform Architecture, Governance, and Environmental Dynamics," Information Systems Research, vol. 21, no. 4, pp. 675-687, 2010.

[14] A. Tiwana, Platform Ecosystems: Aligning Architecture, Governance, and Strategy. Newnes, 2013.

[15] E. Ayiter, "Integrative Art Education in a Metaverse: Ground," Technoetic Arts, vol. 6, no. 1, pp. 41-53, 2008.

[16] D. Tilson, K. Lyytinen, and C. Sorensen, "Research Commentary-Digital Infrastructures: The Missing IS Research Agenda,"Information Systems Research, vol. 21, no. 4, pp. 748-7592010.

[17] J. Recker, R. Lukyanenko, M. Jabbari, B. M. Samuel, and A. Castellanos, "From Representation to Mediation: A New Agenda for Conceptual Modeling Research in a Digital World," MIS Quarterly, 2021.

[18] C. Alaimo and J. Kallinikos, "Computing the Everyday: Social Media as Data Platforms," The Information Society, vol. 33, no. 4, pp. 175-191, 2017.

[19] R. Baskerville, M. Myers, and Y. Yoo, "Digital First: The Ontological Reversal and New Challenges for IS," MIS Quarterly, 2019.

[20] Y. Yoo, "Computing in Everyday Live: A Call for Research on Experiential Computing," MIS Quarterly, Article vol. 34, no. 2, pp. 213-231, 2010.

[21] H. A. Simon, The Sciences of the Artificial. Cambridge, MA: MIT Press, 1996.

[22] S. Seidel, N. Berente, B. Martinez, A. Lindberg, K. Lyytinen, and J. V. Nickerson, "Succeeding with Autonomous Tools in Systems Design: Reflective Practice \& Ubisoft's Ghost Recon Wildlands Project.," IEEE Computer, vol. 51, no. 10, pp. 16-23, 2018.

[23] S. Y. Lee and J. Kang, "Effect of Virtual Reality Meditation on Sleep Quality of Intensive Care Unit Patients: A Randomised Controlled Trial," Intensive and Critical Care Nursing, vol. 59, p. 102849, 2020.

[24] J. Recker, M. Indulska, P. Green, A. Burton-Jones, and R. Weber, "Information Systems as Representations: A Review of the Theory and Evidence," Journal of the Association for Information Systems, vol. 20, no. 6, pp. 736-786, 2019.

[25] A. Burton-Jones, J. Recker, M. Indulska, P. Green, and R. Weber, "Assessing Representation Theory with a Framework for Pursuing Success and Failure," MIS Quarterly, vol. 41, no. 4, 2017.

[26] A. Baird and L. M. Maruping, "The Next Generation of Research on IS Use: A Theoretical Framework of Delegation to and from Agentic IS Artifacts," MIS Quarterly, vol. 45 , no. 1, 2021.

[27] S. Vakulenko, M. de Rijke, M. Cochez, V. Savenkov, and A. Polleres, "Measuring Semantic Coherence of a Conversation," in International Semantic Web Conference, 2018: Springer, pp. 634-651.
[28] A. Stadler and T. H. Kolbe, "Spatio-semantic Coherence in the Integration of 3D City Models," in Proceedings of the 5th International ISPRS Symposium on Spatial Data Quality ISSDQ 2007 in Enschede, The Netherlands, 13-15 June 2007, 2007.

[29] C. Alexander, "A City is Not a Tree," Architectural Forum, vol. 122, pp. 58-62, 1965.

[30] S. Zuboff, In the Age of the Smart Machine: The Future of Work and Power. Basic Books, New York, 1988.

[31] O. Valdez-De-Leon, "How to Develop a Digital Ecosystem: A Practical Framework," Technology Innovation Management Review, vol. 9, no. 8, 2019.

[32] H. Mintzberg, The Structuring of Organizations. A Synthesis of the Research. Prentice Hall, 1979.

[33] C. Goanta, "Selling LAND in Decentraland: The Regime of Non-fungible Tokens on the Ethereum Blockchain Under the Digital Content Directive," in Disruptive Technology, Legal Innovation, and the Future of Real Estate: Springer, 2020, pp. 139-154.

[34] G. Parker, M. Van Alstyne, and X. Jiang. Platform Ecosystems: How Developers Invert the Firm, 2016.

[35] J. V. Nickerson and M. zur Muehlen, "The Ecology of Standards Processes: Insights form Internet Standard Making," MIS Quarterly, Article vol. 30, pp. 467-488, 2006.

[36] F. Bostoen, “Epic v Apple: Antitrust's Latest Big Tech Battle Royale," European Competition and Regulatory Law Review, vol. 5, no. 1, pp. 79-85, 2021.

[37] J. Zittrain, The Future of the Internet-And how to Stop It. London: Allen Lane, 2008.

[38] S. Nambisan, K. Lyytinen, A. Majchrzak, and M. Song, "Digital Innovation Management: Reinventing Innovation Management Research in a Digital World," MIS Quarterly, vol. 41, no. 1, 2017.

[39] H. A. Simon, "The Architecture of Comlexity," Proceedings of the American Philosophical Society, vol. 106, no. 6, pp. 467-482, 1962.

[40] T. Eisenmann, G. Parker, and M. Van Alstyne, "Platform Envelopment," Strategic Management Journal, vol. 32, no. 12, pp. 1270-1285, 2011.

[41] S. Seidel, N. Berente, A. Lindberg, K. Lyytinen, B. Martinez, and J. V. Nickerson, "Artificial Intelligence and Video Game Creation: A Framework for the New Logic of Autonomous Design," Journal of Digital Social Research, vol. 2, no. 3, pp. 126-157, 2020.

[42] S. Mondesire and R. P. Wiegand, "Evolving a Nonplayable Character Team with Layered Learning," in 2011 IEEE Symposium on Computational Intelligence in Multicriteria Decision-Making (MDCM), 2011: IEEE, pp. 52-59. 